\title{
Mode and gain analysis for symmetric and staggered grating-waveguide free-electron laser
}

\author{
Yuan-Yao Lin and Yen-Chieh Huang \\ Institute of Photonics Technologies, Department of Electrical Engineering, National Tsing Hua University, Hsinchu 300, Taiwan
}

(Received 15 October 2006; published 2 March 2007)

\begin{abstract}
A grating-waveguide free-electron laser is driven by an axial electron beam that propagates between two planar gratings. This investigation addresses the mode analysis, gain calculation, and frequency tuning for such a device with arbitrary longitudinal and transverse displacements between the two gratings. It demonstrates that a grating waveguide free-electron laser is a promising coherent $\mathrm{THz}$ emitter when it is adopted as a backward-wave oscillator or a distributed-feedback oscillator.
\end{abstract}

DOI: 10.1103/PhysRevSTAB.10.030701

PACS numbers: 41.60.Cr

\section{INTRODUCTION}

When an electron propagates near the surface of a periodic metal structure, its scattering between the electron field and the metal grating generates electromagnetic radiation. This fact has been extensively exploited to generate microwave radiation. For example, a microwave backward-wave oscillator (BWO) employs an axial electron beam in a circular waveguide with a periodic structure. As the radiation wavelength and thus the radiating device become smaller, a planar structure for the device becomes a preferred geometry due to the ease of fabrication and implementation. For instance, a popular radiation source in the millimeter and submillimeter regime is called the Smith-Purcell radiator [1], in which a stream of electrons traverses close to a planar grating to emit radiation into a semi-open space at an angle that is governed by the radiation wavelength, the grating period, and the electron energy. A so-called planar orotron [2] utilized a planar conductor which is installed above the Smith-Purcell grating to increase the radiation feedback to the electron beam. Killoran et al. [3] further developed a dual-grating resonator in which an electron beam propagates closer to one of the two parallel gratings in the grating-vector direction. The grating that is closer to the electron beam is used to generate the Smith-Purcell radiation and the other is used to reflect radiation of certain angles back toward the electron beam. However, the Smith-Purcell radiation power was typically too low to be considered practical until Urata et al. [4] observed superradiance at $\mathrm{THz}$ frequencies by using a $30-40 \mathrm{keV}$ electron beam from an electron microscope. Several different theories [5-8] have been proposed to explain Urata's observation. However, experiments to reproduce Urata's results and verify the theories are still underway [9]. The radiation power can be increased by promoting beam-wave coupling using a waveguiding structure. Since an electron-driven periodic circular waveguide has been proven successful in the microwave regime, a periodic waveguide formed by two planar gratings can be a simplified and yet promising structure for implementing radiation sources at $\mathrm{THz}$ frequencies. Unlike a BWO in a circular metal enclosure, a grating-waveguide free-electron laser (GWFEL) has the flexibility of an adjustable transverse gap and longitudinal displacement between the two gratings for various tuning purposes. This grating-waveguide structure resembles a BWO in vacuum electronics and resembles a distributed-feedback laser in solid-state photonics. This paper analyzes the guiding mode, radiation gain, and frequency tuning of a GWFEL in the THz-frequency regime, subject to arbitrary longitudinal and transverse displacements between the two gratings.

This paper is organized as follows. Section II utilizes a mode-expansion technique to derive the dispersion relationship for an infinitely long GWFEL with square-wave surface corrugation and adopts the plasma-dielectric approach [10] to develop a general formula for calculating its small-signal gain. A GWFEL that exhibits mirror symmetry about the longitudinal axis is herein called a symmetric GWFEL. Section III discusses a few numerical examples of symmetric GWFELs operated at $\mathrm{THz}$ frequencies. A symmetric GWFEL becomes a staggered GWFEL when one grating is displaced by a half-period in the longitudinal direction. Section IV compares the performance of a staggered GWFEL with that of a symmetric GWFEL. Both longitudinal and transverse adjustments of the relative position of the two gratings improve the frequency tuning of a GWFEL. Section V draws conclusions.

\section{MODE ANALYSIS AND GAIN CALCULATION}

Figure 1 depicts the configuration of a GWFEL. Two lossless conducting gratings with rectangular grooves are arranged in parallel to each other with their grating vectors aligned in the $z$ direction. The two gratings do not have any variation in the $x$ direction and form a waveguide for waves that propagate in the $\pm z$ direction. Each grating has a period $\Lambda_{g}$, a groove depth $D$, and a groove width $W$. The gap between the two gratings is $G$. To study this device with a general configuration, one grating is allowed to shift in the longitudinal direction by an arbitrary distance $d$ from the other grating. The top and bottom grating surfaces can be described by 


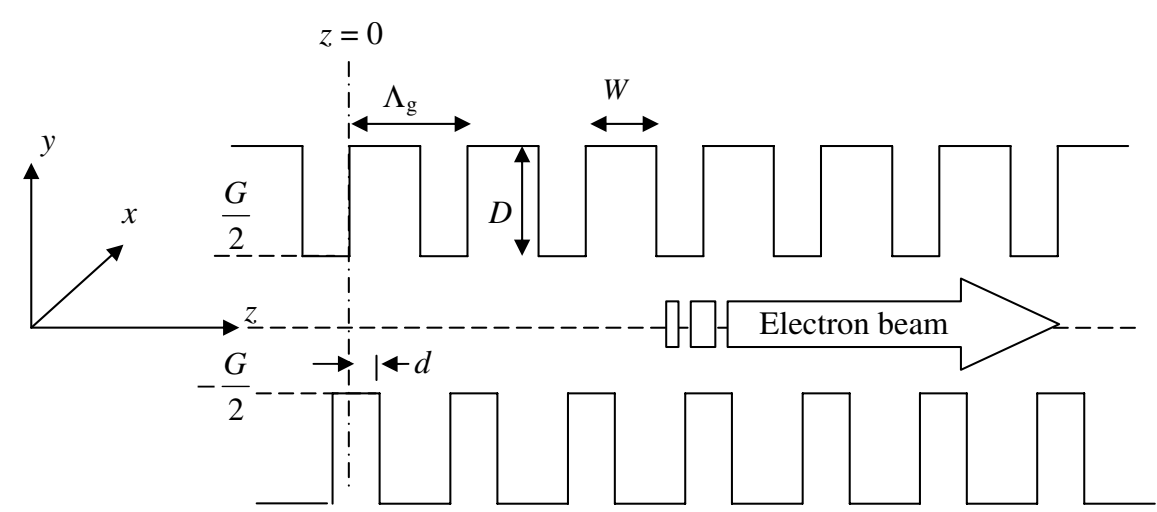

FIG. 1. The schematic of a GWFEL. The symbols $\Lambda_{g}, D, W$, and $G$ are the grating period, groove depth, groove width, and grating gap, respectively. In our analysis, one of the gratings can be displaced from the other by a longitudinal distance $d$. $G$ is also adjustable. The electron beam propagates in $z$. The structure has no variation in $x$.

$$
y_{t}(z)= \begin{cases}\frac{G}{2}+D, & 0 \leq z<W \\ \frac{G}{2}, & W \leq z<\Lambda_{g}\end{cases}
$$

and

$$
y_{b}(z)= \begin{cases}-\frac{G}{2}-D, & d \leq z<d+W \\ -\frac{G}{2}, & d+W \leq z<\Lambda_{g}+d,\end{cases}
$$

respectively, where the subscripts $t$ and $b$ denote the top and bottom boundaries, respectively, and the periodic boundary condition $y_{t, b}\left(z+\Lambda_{g}\right)=y_{t, b}(z)$ is satisfied along $z$.

The analysis herein assumes that a uniform electron beam propagates in the $z$ direction and fills up the waveguide-gap region defined by $-G / 2 \leq y \leq G / 2$. This moving plasma can be modeled by a dielectric constant associated with each waveguide mode, as will be shown below. Since the electron energy is coupled to the waveguide modes through longitudinal electric fields, only the transverse-magnetic (TM) waves in the waveguide region are considered. In the guiding region $-G / 2 \leq y \leq$ $G / 2$, the complex axial electric-field intensity $E_{z}$ can be expanded as

$$
\begin{aligned}
E_{z}= & \sum_{p=-\infty}^{\infty}\left\{E_{p}^{b} \sinh \left[\alpha_{p}\left(\frac{G}{2}-y\right)\right] \exp \left(j \phi_{p}\right)\right. \\
& \left.+E_{p}^{t} \sinh \left[\alpha_{p}\left(\frac{G}{2}+y\right)\right]\right\} \exp \left(-j p \mathrm{~K}_{g} z\right) \\
& \times \exp \left[j\left(\omega t-k_{z} z\right)\right]
\end{aligned}
$$

and the corresponding $x$-component magnetic-field intensity $H_{x}$ is given by

$$
\begin{aligned}
H_{x}= & \sum_{p=-\infty}^{\infty}\left\{H_{p}^{b} \cosh \left[\alpha_{p}\left(\frac{G}{2}-y\right)\right] \exp \left(j \phi_{p}\right)\right. \\
& \left.+H_{p}^{t} \cosh \left[\alpha_{p}\left(\frac{G}{2}+y\right)\right]\right\} \exp \left(-j p \mathrm{~K}_{g} z\right) \\
& \times \exp \left[j\left(\omega t-k_{z} z\right)\right],
\end{aligned}
$$

where $p$ is the index of the spatial harmonics, $k_{z}$ is the wave number in the $z$ direction for $p=0, \mathrm{~K}_{g}=2 \pi / \Lambda_{g}$ is the grating wave number, and $\omega$ is the angular frequency of the wave. The phase introduced by the longitudinal displacement, $d$, between the two grating halves is defined as

$$
\phi_{p}=\left(k_{z}+p \mathrm{~K}_{g}\right) d .
$$

From Helmholtz's equation, the field decay rate of the $p$ th mode in the $y$ direction $\alpha_{p}$ satisfies

$$
\alpha_{p}^{2}=\left(k_{z}+p \mathrm{~K}_{g}\right)^{2}-\frac{\omega^{2}}{c^{2}\left(1+\chi_{p}^{\prime}\right)},
$$

where $c$ is the wave speed in vacuum, and $\varepsilon_{p}=1+\chi_{p}^{\prime}$ is the dielectric constant of the plasma dielectric with $\chi_{p}^{\prime}$ being the plasma susceptibility associated with the $p$ th waveguide mode in the electron rest frame. The specific expression of $\chi_{p}^{\prime}$ was derived elsewhere in [10], and is given by

$$
\chi_{p}^{\prime}=-\frac{\omega_{p l}^{2}}{\gamma^{3}\left[\omega-c \beta \cdot\left(k_{z}+p \mathrm{~K}_{g}\right)\right]^{2}},
$$

where $\beta$ is electron's longitudinal speed normalized to $c$, and $\omega_{p l}$ is the plasma frequency in the laboratory frame

$$
\omega_{p l}^{2}=\frac{16 c^{2}}{\beta d_{x} d_{y}} \frac{I_{e}}{I_{A}}
$$

with $I_{A}$ the Alfvén current, $I_{e}$ the electron-beam current, and $d_{x}$ and $d_{y}$ the electron beam diameters in the $x$ and $y$ directions, respectively. Under the synchronism condition $\omega-c \beta\left(k_{z}+p \mathrm{~K}_{g}\right)=0$ the electric susceptibility, given by Eq. (7), diverges. Generally, $\alpha_{p}$ can be real or imaginary as long as the expanded fields satisfy the boundary conditions. To simplify the calculations, the sign of $\alpha_{p}$ is lumped into the mode-field coefficient and the sign of $\alpha_{p}$ is set to be always positive. 
In the groove region of the top grating $G / 2 \leq y \leq$ $(G / 2+D)$, the $z$-component electric field can be expanded as

$$
E_{z}= \begin{cases}\sum_{n=0}^{\infty} & \bar{E}_{n}^{t} \cos \left(\frac{n \pi z}{W}\right) \frac{\sinh \left[\kappa_{n}\left(y-\frac{G}{2}-D\right)\right]}{\cosh \left(\kappa_{n} D\right)} \exp (j \omega t) \quad 0 \leq z \leq W \\ 0 & \text { otherwise, }\end{cases}
$$

and the corresponding $x$-component magnetic field in the same region is given by

$$
H_{x}=\sum_{n=0}^{\infty} \bar{H}_{n}^{t} \cos \left(\frac{n \pi z}{W}\right) \frac{\cosh \left[\kappa_{n}\left(y-\frac{G}{2}-D\right)\right]}{\sinh \left(\kappa_{n} D\right)} \exp (j \omega t),
$$

where $\bar{E}_{n}^{t}$ and $\bar{H}_{n}^{t}$ are the expansion coefficients for the $n$th spatial harmonic of the electric and magnetic fields, re- spectively. The boundary condition on the conducting surface requires that $E_{z}$ vanishes $y=G / 2+D$ and $E_{y}=$ $-(j / \omega \varepsilon)\left(\partial H_{x} / \partial z\right)$ vanishes at both $z=0$ and $z=W$. Also, for a periodic structure, $E_{i}\left(z+\Lambda_{g}\right)=$ $\exp \left(-j k_{z} \Lambda_{g}\right) E_{i}(z)$ and $H_{i}\left(z+\Lambda_{g}\right)=\exp \left(-j k_{z} \Lambda_{g}\right) H_{i}(z)$ with $i=x, y, z$ must hold for a periodic structure. From the homogeneous Helmholtz equation, the coefficient $\kappa_{n}$ satisfies

$$
\kappa_{n}^{2}=\left(\frac{n \pi}{W}\right)^{2}-\frac{\omega^{2}}{c^{2}}
$$

Likewise, in the groove region of the bottom grating, $-(G / 2+D) \leq y \leq G / 2$, the axial electric field and the corresponding magnetic field in $x$ are

$$
E_{z}= \begin{cases}\sum_{n=0}^{\infty} \bar{E}_{n}^{b} \cos \left(\frac{n \pi(z-d)}{W}\right) \frac{\sinh \left[\kappa_{n}\left(y+\frac{G}{2}+D\right)\right]}{\cosh \left(\kappa_{n} D\right)} \exp (j \omega t), & d \leq z \leq W+d, \\ 0, & \text { otherwise }\end{cases}
$$

and

$$
H_{x}=\sum_{n=0}^{\infty} \bar{H}_{n}^{b} \cos \left(\frac{n \pi(z-d)}{W}\right) \frac{\cosh \left[\kappa_{n}\left(y+\frac{G}{2}+D\right)\right]}{\sinh \left(\kappa_{n} D\right)} \exp (j \omega t),
$$

respectively.

After matching all of the mode fields at the boundaries $y=G / 2$ and $y=-G / 2$, one can obtain the characteristic equation for the guiding modes in the grating-waveguide structure. According to the derivations in Appendix A, the characteristic equation for the guiding wave can be approximated by the equation

$$
b_{00} \pm\left|a_{00}\right|=1
$$

where

$$
\begin{aligned}
\left|a_{00}\right|= & |\bar{\omega} \bar{W} \tan (2 \pi \bar{\omega} \bar{D})|\left\{\left[\sum_{p=-\infty}^{\infty} \frac{\left(1+\delta_{p 0} \chi^{\prime}\right)}{\bar{\alpha}_{p} \sinh \left(2 \pi \bar{\alpha}_{p} \bar{G}\right)} \cos (2 \pi p \bar{d}) \operatorname{sinc}^{2}\left[\left(p+\bar{k}_{z}\right) \bar{W}\right]\right]^{2}\right. \\
& \left.+\left[\sum_{p=-\infty}^{\infty} \frac{\left(1+\delta_{p 0} \chi^{\prime}\right)}{\bar{\alpha}_{p} \sinh \left(2 \pi \bar{\alpha}_{p} \bar{G}\right)} \sin (2 \pi p \bar{d}) \operatorname{sinc}^{2}\left[\left(p+\bar{k}_{z}\right) \bar{W}\right]\right]^{2}\right\}^{1 / 2}
\end{aligned}
$$

and

$$
\begin{aligned}
b_{00}= & \bar{\omega} \bar{W} \tan (2 \pi \bar{\omega} \bar{D}) \sum_{p=-\infty}^{\infty} \frac{1+\delta_{p 0} \chi_{p}^{\prime}}{\bar{\alpha}_{p} \sinh \left(2 \pi \bar{\alpha}_{p} \bar{G}\right)} \\
& \times \cosh \left(2 \pi \bar{\alpha}_{p} \bar{G}\right) \operatorname{sinc}^{2}\left[\left(p+\bar{k}_{z}\right) \bar{W}\right]
\end{aligned}
$$

with the dimensionless variables defined as $\bar{D} \equiv D / \Lambda_{g}$, $\bar{W} \equiv W / \Lambda_{g}, \bar{G} \equiv G / \Lambda_{g}, \bar{d} \equiv d / \Lambda_{g}, \bar{\alpha}_{p} \equiv \alpha_{p} / \mathrm{K}_{g}, \bar{\omega}=$ $\omega /\left(c K_{g}\right)$. As stated in Eqs. (A15) and (A16), the coefficients $a_{n m}$ are related to the coupling strength of the fields between the top and bottom gratings. Accordingly, unlike $b_{00}$, the coefficient, $a_{00}$, is strongly influenced by the waveguide gap $\bar{G}$. When $\bar{G}$ is increased, the amplitude of $\left|a_{00}\right|$ declines rapidly. The \pm signs in Eq. (14) correspond to two sets of distinct mode profiles in the transverse direction, as will be shown in the next section. Equation (14) describes the dispersion characteristics of the electromagnetic modes in an infinitely long gratingwaveguide structure in the presence of an electron beam. The following assumes that Eq. (14) is sufficiently accurate for studying the gain per unit length of a long enough GWFEL. The radiation gain from the electron beam is embedded in the dielectric constant of the moving plasma in the waveguide region or in the term $\delta_{p 0} \chi_{p}^{\prime}=\chi_{0}^{\prime}$. The perturbation technique that was described by Andrews et $a l$. for a Smith-Purcell FEL [5] is applied herein to expand the dispersion relation (14) about the beam-wave synchronous point $\omega_{0}=\beta c k_{0}$ : 


$$
\left(\delta \omega-\beta_{g} c \delta k\right)(\delta \omega-\beta c \delta k)^{2}=\frac{\omega_{p l}^{2}}{\gamma^{3}} \frac{S\left(\omega_{0}, k_{0}\right)}{R_{\omega}\left(\omega_{0}, k_{0}\right)},
$$

where $\beta_{g} c$ is the group velocity of the wave, $\delta \omega=\omega-\omega_{0}, \delta k=k_{z}-k_{0}$, and $R_{\omega}=\partial R /\left.\partial \omega\right|_{\omega=\omega_{0}, k_{z}=k_{0}}$ with $R$ and $S$ expressed by

$$
R=\bar{\omega} \bar{W} \tan (2 \pi \bar{\omega} \bar{D})\left\{\sum_{p=-\infty}^{\infty} \frac{\cosh \left(2 \pi \bar{\alpha}_{p} \bar{G}\right)}{\bar{\alpha}_{p} \sinh \left(2 \pi \bar{\alpha}_{p} \bar{G}\right)} \operatorname{sinc}^{2}\left[\left(p+\bar{k}_{z}\right) \bar{W}\right] \pm\left|\sum_{p=-\infty}^{\infty} \frac{e^{i \phi_{p}}}{\bar{\alpha}_{p} \sinh \left(2 \pi \bar{\alpha}_{p} \bar{G}\right)} \operatorname{sinc}^{2}\left[\left(p+\bar{k}_{z}\right) \bar{W}\right]\right|\right\}
$$

and

$$
S=\bar{\omega} \bar{W} \tan (2 \pi \bar{\omega} \bar{D}) \frac{\cosh \left(2 \pi \bar{\alpha}_{p} \bar{G}\right) \pm 1}{\bar{\alpha}_{0} \sinh \left(2 \pi \bar{\alpha}_{0} \bar{G}\right)} \operatorname{sinc}^{2}\left(\bar{k}_{z} \bar{W}\right) .
$$

The imaginary parts of $\delta \omega$ and $\delta k$ cause the mode fields to grow or decay exponentially, and represent the instability of this device. The next two sections will continue the discussion of this instability in different operation regimes for specific device configurations.

\section{SYMMETRIC GRATING-WAVEGUIDE FEL}

In our earlier analysis, we have kept an arbitrary longitudinal displacement $\bar{d}$ between the two gratings. When $\bar{d}$ is set to zero, the top grating is the mirror image of the bottom grating with respect to the $y=0$ plane. This structure is called a symmetric GWFEL herein. At $\bar{d}=0$, the dispersion relation (14) becomes

$$
\bar{\omega} \bar{W} \tan (2 \pi \bar{\omega} \bar{D}) \sum_{p=-\infty}^{\infty} \frac{1+\delta_{p 0} \chi_{p}^{\prime}}{\tilde{\alpha}_{p}} \operatorname{coth}\left(\pi \bar{\alpha}_{p} \bar{G}\right) \operatorname{sinc}^{2}\left[\left(p+\bar{k}_{z}\right) \bar{W}\right]=1,
$$

and

$$
\bar{\omega} \bar{W} \tan (2 \pi \bar{\omega} \bar{D}) \sum_{p=-\infty}^{\infty} \frac{1+\delta_{p 0} \chi_{p}^{\prime}}{\tilde{\alpha}_{p}} \tanh \left(\pi \bar{\alpha}_{p} \bar{G}\right) \operatorname{sinc}^{2}\left[\left(p+\bar{k}_{z}\right) \bar{W}\right]=1
$$

The dispersion equation (20) yields to an axial electric field with even symmetry about the $y=0$ plane. Specifically the axial field is given by

$$
E_{z}=\sum_{p=-\infty}^{\infty} H_{p}^{t} \sinh \left(\alpha_{p} \frac{G}{2}\right) \cosh \left(\alpha_{p} y\right) \exp \left(-j p \mathrm{~K}_{g} z\right) \exp \left[j\left(\omega t-k_{z} z\right)\right]
$$

In the following, the guiding mode associated with this field is called an even mode. Equation (21) leads to an $E_{z}$ with odd symmetry about the $y=0$ plane, which is given by

$$
E_{z}=\sum_{p=-\infty}^{\infty} E_{p}^{t} \cosh \left(\alpha_{p} \frac{G}{2}\right) \sinh \left(\alpha_{p} y\right) \exp \left(-j p \mathrm{~K}_{g} z\right) \exp \left[j\left(\omega t-k_{z} z\right)\right]
$$

In the following, the guiding mode associated with this field is called an odd mode. In practice, an electron beam is symmetric about the longitudinal axis and is strongly coupled to the longitudinal electric field with even symmetry in $y$. Indeed, our numerical calculations indicate that odd guiding modes have a much lower gain because they have a zero longitudinal field on the axis. In the following, the odd modes are neglected and only the even modes are considered in GWFEL. However, we note here that insertion of a conducting plate in the $x-z$ plane at $y=0$ does not change the odd-mode profile in the region $y>0$ or $y<0$. As a result, Eq. (23) for $y>0$ or $y<0$ also describes the axial mode field of a planar orotron [2]. At THz frequencies, the electron aperture is usually small in the guiding structure. If the waveguide gap is mostly filled with electrons, then the asymmetry of the mode fields due to the asymmetry of the orotron structure could deflect electrons in the transverse direction.

In the absence of an electron beam, the electronic susceptibility $\chi_{p}{ }^{\prime}$ is zero in Eq. (21). Figure 2 shows the dispersion or the band diagram for the even modes in a cold structure (with $\chi_{p}{ }^{\prime}=0$ ) with $\bar{W}=0.6, \bar{D}=1.2$, and $\bar{G}=3$. Unlike the surface modes in a Smith-Purcell FEL [10], the guiding modes in a GWFEL have high-frequency bands due to transverse resonances in a grating waveguide. The condition $\vec{k}_{z}=0.5 \times m$ with $m=1,2,3, \ldots$ is the Bragg resonance condition for a longitudinal grating. Whenever $\bar{k}_{z}=0.5 \times m$ is satisfied, the space harmonics 


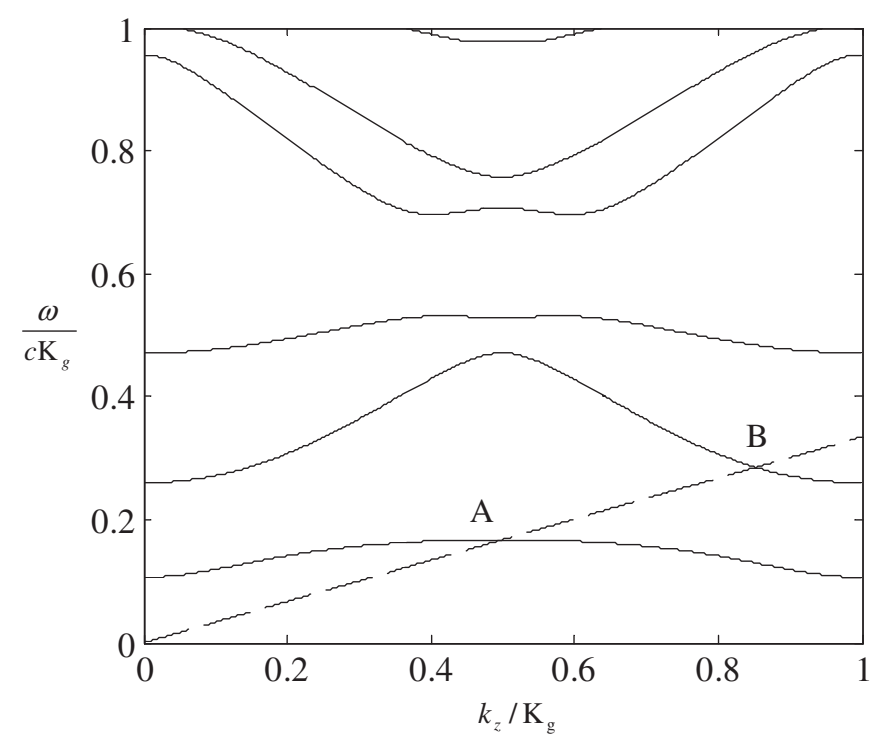

FIG. 2. The dispersion curves of a grating waveguide with $\bar{W}=0.6, \bar{D}=1.2, \bar{G}=3$, and $\bar{d}=0$. A $31.36 \mathrm{keV}$ beam line (dashed line) intercepts the lowest dispersion curve at the first Bragg resonance (point $\mathrm{A}$ ) and the second curve at a point having a negative slope (point B). Point A and point B could result in distributed-feedback oscillation and backward-wave oscillation, respectively, as long as the driving current exceeds a threshold value.

of the mode field are paired up to form a standing wave in the axial direction of the grating waveguide, which results in the zero slope or zero group velocity at $\bar{k}_{z}=0.5 \times \mathrm{m}$ in Fig. 2. An electron beam line with a slope $\beta$ can be drawn on the same plot to intercept the dispersion curves at the beam-wave synchronous points. The high-frequency bands, when intercepted by a beam line, offer the opportunity of generating high-frequency radiations. For example, given a $30-50 \mathrm{keV}$ beam and a $50 \mu \mathrm{m}$ grating period, the synchronous wavelength varies from 298 to $469 \mu \mathrm{m}$ in the first (lowest-frequency) dispersion curve and from 167 to $183 \mu \mathrm{m}$ in the second dispersion curve in Fig. 2.

Figure 3 plots the on-axis $E_{z}$ field against $z$ at the $m=1$ Bragg resonance $\bar{k}_{z}=0.5$ for the first dispersion curve in Fig. 2. The structure parameters for the plot are $\bar{W}=0.6$, $\bar{D}=1.2$, and $\bar{G}=1$. The bright and dark shaded areas represent the groove and tooth of the grating, respectively, along $z$. As expected from the Bragg condition $\bar{k}_{z}=0.5$, the $E_{z}$ field has a standing-wave profile with a period of $2 \Lambda_{g}$, which is similar to the acceleration field of a standing-wave accelerator operating in the $\pi$ mode. The standing-wave nodes and antinodes coincide with the longitudinal centers of the teeth and the grooves, respectively. The inset of Fig. 3 plots the $E_{z}$ field profile versus $y$ at $z / \Lambda_{g}=0.3$ or at the longitudinal center of a groove. The field extends into the two grooves and vanishes at the top and bottom conducting boundaries $y= \pm(G / 2+D)$, as expected.

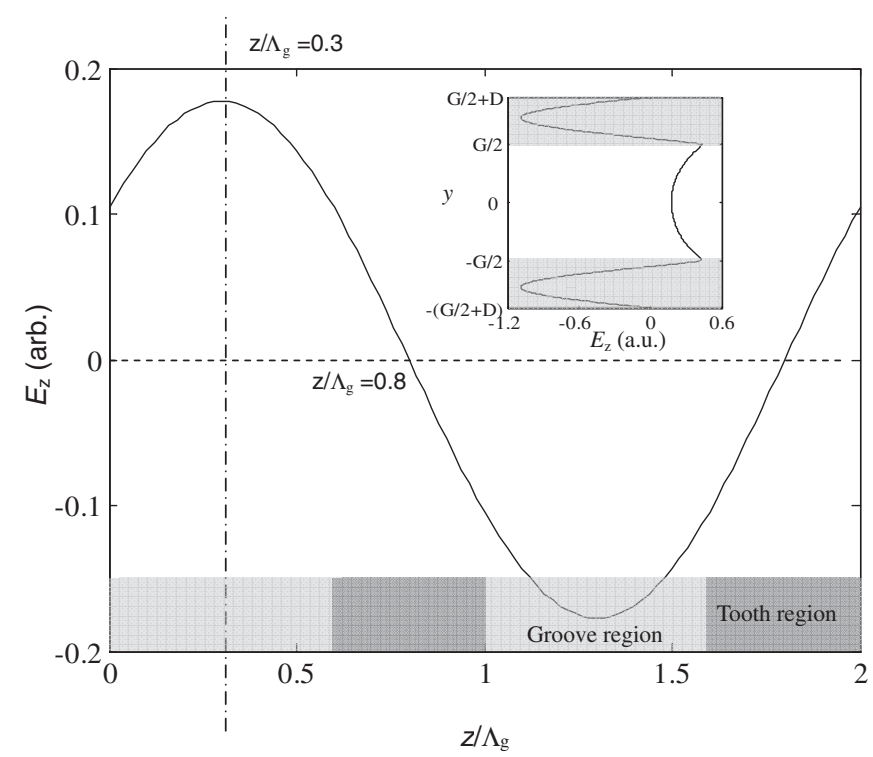

FIG. 3. $E_{z}$ field along the $z$ axis of a symmetric grating waveguide for $\bar{k}_{z}=0.5$. The bright and dark areas above the horizontal axis mark the groove and tooth of the grating, respectively, along $z$. The inset illustrates the $E_{z}$ field profile along $y$ at $z / \Lambda_{g}=0.3$ or at the center of a groove.

When a GWFEL is used as an amplifier, the small signal gain of such an amplifier in the steady state can be calculated by setting $\delta \omega=0$ in Eq. (17) and solving for the imaginary part of $\delta k$ to obtain the gain coefficient $g=$ $\left.\operatorname{Im}(\delta k)\right|_{\delta \omega=0}$. Setting $\bar{d}=0$ in Eq. (17) yields the gain coefficient $g$ of a symmetric GWFEL as a function of the electron-beam energy in Fig. 4, for a beam current of $5 \mathrm{~mA}$ and beam diameters of $d_{x}=d_{y}=150 \mu \mathrm{m}$. The continuous and dashed lines are the gain curves that correspond to the first and second dispersion curves in Fig. 2, respectively. Equation (17) shows that the gain coefficient $g=$ $\left.\operatorname{Im}(\delta k)\right|_{\delta \omega=0}$ diverges at points of zero group velocity or at the Bragg resonances. At the resonant wavelength, the distributed feedback from the grating structure adds up coherently, resulting in an infinite value for the FEL gain. As displayed in Fig. 4, the electron beams with energies of 31.36 and $2.95 \mathrm{keV}$ can drive the Bragg resonances at $\bar{k}_{z}=$ 0.5 and 1 in the first dispersion curve to produce radiations with wavelengths of 298 and $469 \mu \mathrm{m}$, respectively. Since the slope of the beam line is always less than unity or $\beta<$ 1 , the wave cannot resonate at $\bar{k}_{z}=0.5$ in the second dispersion curve for this particular set of design parameters. However, the $\bar{k}_{z}=1$ Bragg resonance is available in the second dispersion curve at a beam energy of $18.6 \mathrm{keV}$, yielding a radiation wavelength of $183 \mu \mathrm{m}$. Accordingly, the high-frequency dispersion curves are quite useful for generating short-wavelength radiation from a low-energy electron beam that is synchronous to a Bragg resonance. In practice, a finite-length FEL structure has a finite material loss and a finite output-coupling loss. The finite material loss per unit length is associated with a propagating wave. 


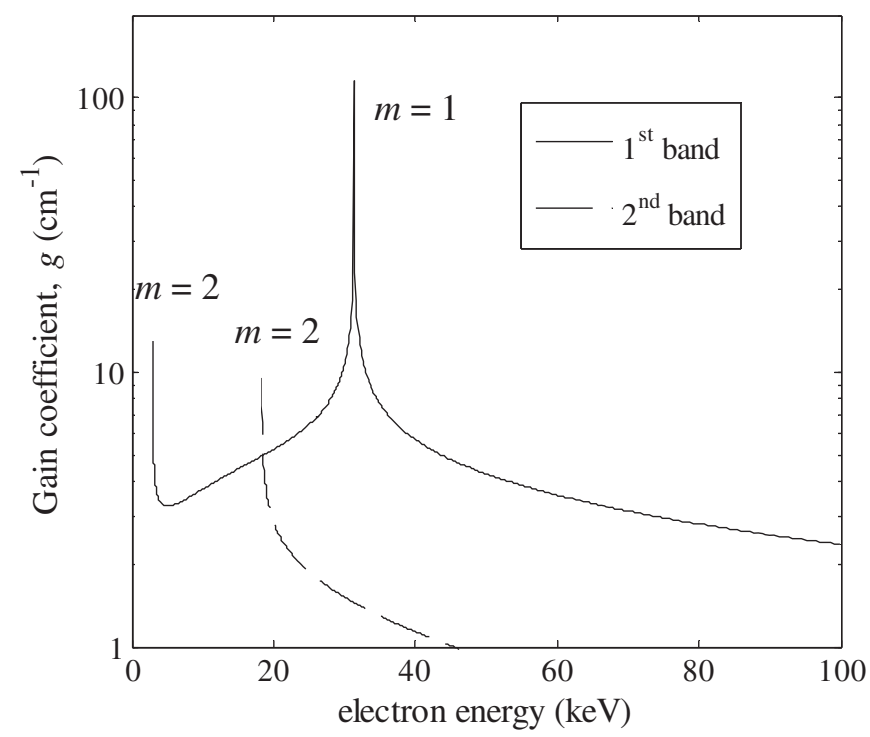

FIG. 4. Small-signal gain coefficient versus electron energy synchronized to the 1st (continuous line) and 2nd (dashed line) dispersion band curves in Fig. 2. The driving current in this calculation is $5 \mathrm{~mA}$ uniformly distributed over beam diameters of $d_{x}=d_{y}=150 \mu \mathrm{m}$. The gain coefficient diverges at the Bragg resonances.

A finite loss coefficient that is associated with each of the two counterpropagating standing-wave components at the Bragg resonance can be used to derive a threshold gain for a distributed-feedback laser during signal buildup [11]. Although this distributed-feedback resonance was recently identified for a Smith-Purcell FEL [12], a complete model for a distributed-feedback GWFEL is yet to be established.

Away from the Bragg resonances, the positive-slope region of the dispersion curve indicates that electromagnetic energy is propagating in the $+z$ direction, whereas the negative-slope region indicates backward-propagating electromagnetic energy. The former is commonly seen in a traveling-wave tube (TWT), whereas the latter is known to occur in a BWO for microwave generation. The proposed planar structure of a GWFEL not only has the potential to achieve low-threshold mirrorless oscillation near the Bragg resonance but also permits the implementation of a TWT or a BWO at THz frequencies. Swegle [13] and Andrews et al. [5] derived the threshold gain for a BWO $g_{t h}=$ $\xi_{0} \sqrt{3} /(2 L)$, where $\xi_{0}=1.97$ and $L$ is the length of the BWO. For example, the BWO threshold gain for a $5 \mathrm{~cm}$ long GWFEL is $0.34 \mathrm{~cm}^{-1}$, which is everywhere lower than the gain curves in Fig. 4. Therefore the $5 \mathrm{~mA}$ current used for calculating the gain curves is sufficient for establishing backward-wave oscillation in the THz GWFEL. The electromagnetic loss in a metal grating will certainly increase the threshold current of a GWFEL. Just like any other oscillators, such a BWO GWFEL must have enough driving current to overcome both the coupling loss and the material loss.

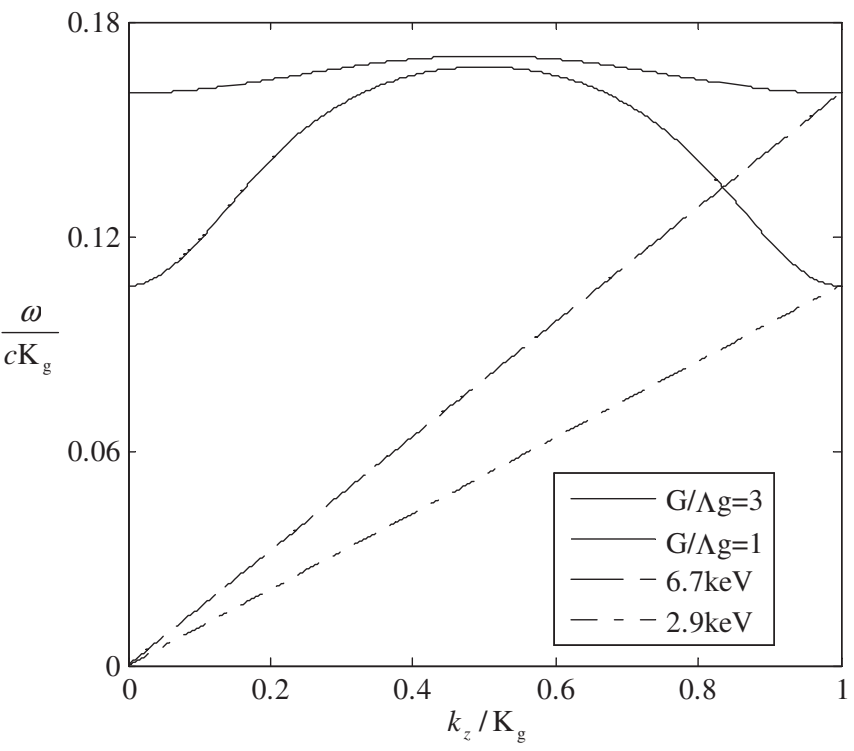

FIG. 5. Illustration of frequency tuning by adjusting the waveguide gap. When the waveguide gap is adjusted from $G / \Lambda g=3$ to 1 , the first dispersion curve (continuous line) in Fig. 2 is changed to the dotted line. Varying the electron energy from 2.9 (dash-dotted line) to $6.7 \mathrm{keV}$ (dashed line) can correspondingly tune the frequency from $\bar{\omega}=0.1$ to 0.16 at the $m=2 \mathrm{Bragg}$ resonance.

A typical BWO adopts a circular enclosure as its waveguide, which does not offer the flexibility of structural tuning. However, a GWFEL employs two parallel gratings as its waveguide, which can be displaced from each other. Figure 5 plots the first dispersion curves of a symmetric grating waveguide for $\bar{W}=0.6, \bar{D}=1.2$, and $\bar{G}=1$ (solid line) and 3 (dotted line). Varying the waveguide gap or $\bar{G}$ tunes the dispersion curve and thus the mode frequency. The radiation frequency from a distributedfeedback oscillator in solid-state photonics is typically fixed by the period of its structure, and cannot be adjusted easily. A GWFEL does not have such a constraint. For instance, if frequency tuning is desired at the Bragg resonance $\bar{k}_{z}=1$, the electron energy can be varied from $2.9 \mathrm{keV}$ (dash-dotted line) to $6.7 \mathrm{keV}$ (dashed line) and the waveguide gap simultaneously adjusted from $\bar{G}=3$ and 1 to tune the frequency from $\bar{\omega}=0.1$ to 0.16 , correspondingly.

Like the waveguide gap $\bar{G}$, the longitudinal displacement $\bar{d}$ of the two grating halves can also be adjusted to vary the dispersion curve and thereby tune the radiation frequency. The following section discusses the effect of $\bar{d}$ on the dispersion relationship and the gain of a GWFEL.

\section{STAGGERED GRATING-WAVEGUIDE FEL}

The proposed GWFEL adopts two planar gratings that can be flexibly shifted from each other in an application. A microwave TWT or BWO generally does not have this flexibility because the enclosure of the waveguide structure 
is circular. This section elucidates the effects of the relative shift of the two gratings in the $z$ direction.

Previously we have derived the mode fields in a grating waveguide with an arbitrary longitudinal displacement $\bar{d}$. The maximum extent of the longitudinal displacement between the two gratings is half of the grating period, or $\bar{d}=0.5$. For such a staggered grating waveguide, the coupling coefficient $\left|a_{00}\right|$ in Eq. (A22) is reduced to

$$
\begin{aligned}
\left|a_{00}\right|= & |\bar{\omega} \bar{W} \tan (2 \pi \bar{\omega} \bar{D})| \sum_{p=-\infty}^{\infty}(-1)^{p}\left(1+\delta_{p 0} \chi_{p}^{\prime}\right) \\
& \times \frac{\operatorname{sinc}^{2}\left[\left(p+\bar{k}_{z}\right) \bar{W}\right]}{\bar{\alpha}_{p} \sinh \left(2 \pi \bar{\alpha}_{p} \bar{G}\right)}
\end{aligned}
$$

Substituting Eq. (24) into Eq. (14) and setting $\chi_{p}^{\prime}=0$ yields Fig. 6(a) which plots the dispersion relationship of a cold staggered grating waveguide with $\bar{W}=0.6, \bar{D}=$ $1.2, \bar{G}=3$, and $\bar{d}=0.5$. Symmetry breaking prevents any pure odd or even mode in such a waveguide. For comparison, Fig. 6(b) plots the dispersion curves of a cold, symmetric waveguide with the same structural dimensions. The continuous lines $(A, C)$ and dashed lines $(B, D)$ in Fig. 6(b) are the even- and odd-mode curves, respectively, in the symmetric waveguide. The mode curves $A^{\prime}-D^{\prime}$ in Fig. 6(a) can be considered to be evolved from curves $A-D$, respectively, when the longitudinal position of the two grating halves are adjusted from $\bar{d}=0$ to $\bar{d}=0.5$. Interestingly, the dispersion curves of the staggered grating waveguide are no longer symmetric about the lines $\bar{k}_{z}=$ $m / 2$, where $m$ is an odd integer. In addition, the zero- group-velocity points in the staggered grating waveguide are also slightly detuned from the odd-order Bragg resonances. The amount of detuning, as derived in Appendix B, depends strongly on the waveguide gap and the mode frequency. When the waveguide gap becomes smaller than the wavelength, the staggering of the grating waveguide appears to double the spatial frequency of the structure in the longitudinal direction. Consequently, the mode fields see an effective structure period that is half of the grating period. The reduced structural period detunes the odd-order Bragg resonances, but preserves the even-order Bragg resonances. For large $\bar{G}$ and $\bar{\omega}$, the detuning near the odd-order Bragg resonances becomes less obvious, because the energy coupling between the two grating halves is weaker and the period of the structure approaches the period of the grating. Figure 7(a) plots the axial field $E_{z}$ versus $z$ at $\bar{k}_{z}=0.5$ in a staggered grating waveguide with the same structure parameters as those for plotting Fig. 6(a). The corresponding tooth and groove regions are drawn on the same plot as shaded boxes. Although a small detuning occurs at $\bar{k}_{z}=0.5$, the period of the $E_{z}$ field is still very close to that of a $\pi$-mode field. The inset in Fig. 7(a) plots $E_{z}$ versus $y$ at $z=0.55 \Lambda_{g}$, which, as expected, vanishes at the conducting surfaces of the grating substrates. Since the $E_{z}$ field decays toward the center of the waveguide gap, the mode field in a staggered grating waveguide can be considered as a linear sum of the two evanescent mode fields on the two grating surfaces, except in that there is a phase shift between the two surface modes due to the longitudinal displacement $\bar{d}$. Although the $E_{z}$

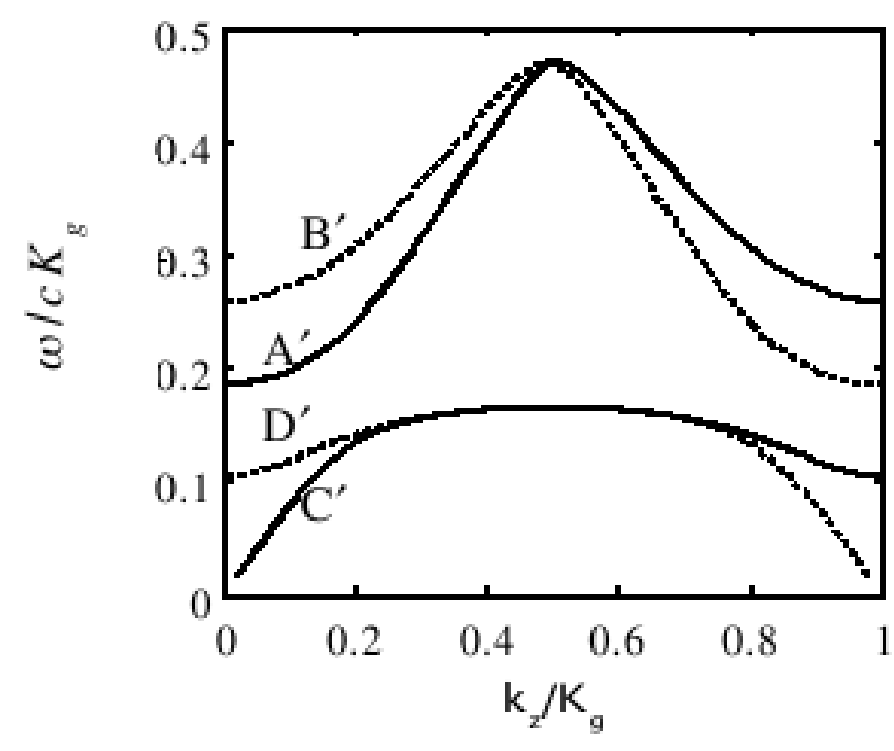

(a)

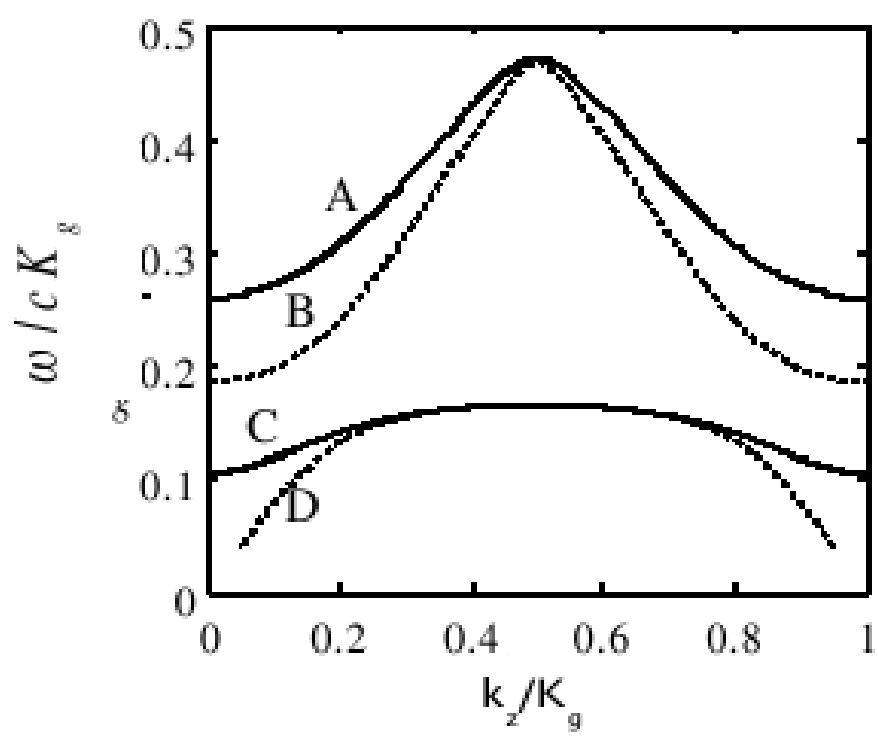

(b)

FIG. 6. (a) Dispersion curves of a staggered grating waveguide with $\bar{W}=0.6, \bar{D}=1.2, \bar{G}=3$, and $\bar{d}=0.5$. (b) Dispersion curves of a symmetric grating waveguide with the same structure parameters except $\bar{d}=0$. Continuous and dashed lines designate even- $E_{z}$ and odd- $E_{z}$ mode curves in (b). The curves $A^{\prime}-D^{\prime}$ in (a) can be considered to be evolved from curves $A-D$ in (b) when $\bar{d}$ is changed from 0 to 0.5 . The dispersion curves in the staggered waveguide are asymmetric about the $m=1$ Bragg resonance. 

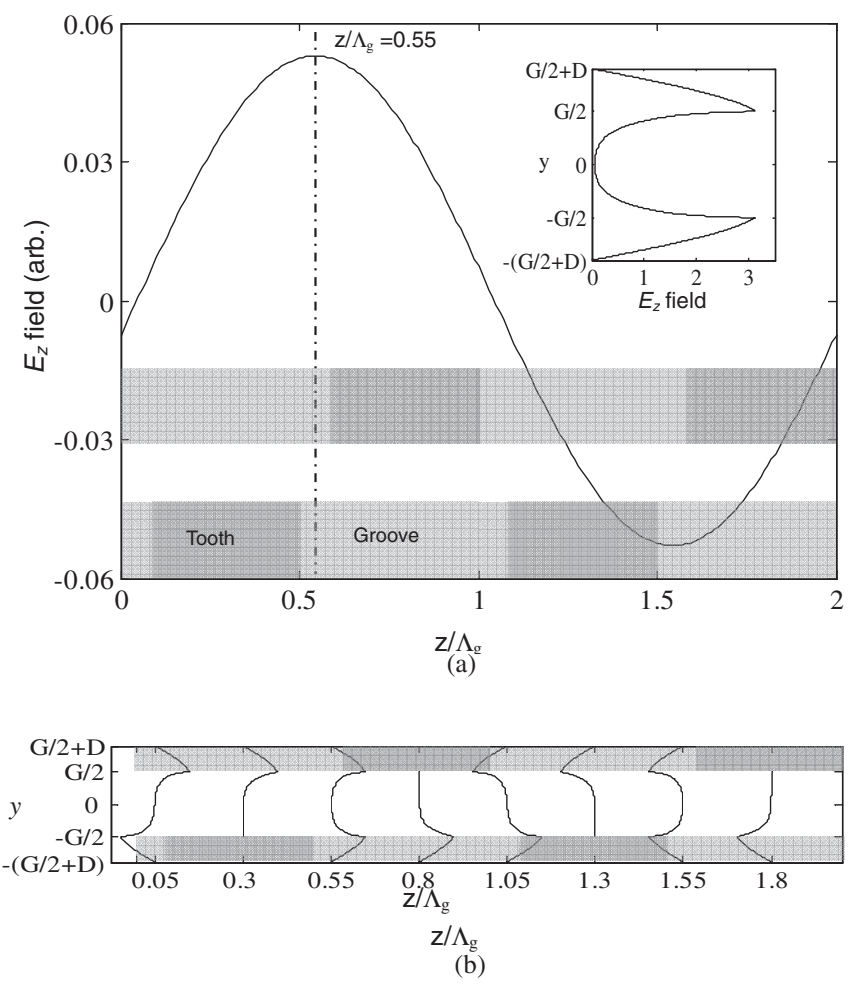

FIG. 7. (a) $E_{z}$ field versus $z$ for $\bar{k}_{z}=0.5$ in a staggered grating waveguide with the same structure parameters used in Fig. 6(a). The brightness and dark areas mark the groove and tooth of the grating, respectively. The inset shows the $E_{z}$ profile versus $y$ at $z / \Lambda_{g}=0.55$. (b) $E_{z}$ field profiles versus $y$ at several $z$ locations in the staggered grating waveguide. The field profile is not a constant but varies along $z$, resulting from the interference of the two surface guiding modes on the two gratings.

field at $z=0.55 \Lambda_{g}$ is an even function of $y$, the field profile varies in the $z$ direction as presented in Fig. 7(b). This field variation is caused by the coupling phase between the electromagnetic energies that are guided by the two grating surfaces.

The small-signal gain of a staggered GWFEL can be computed by using the same technique as used for a symmetric GWFEL, except that $\bar{d}=0.5$ is used in Eq. (17). Figure 8 compares the gain coefficients between a staggered GWFEL and a symmetric GWFEL for a synchronous electron-beam energy of between 0 and $100 \mathrm{keV}$. The structural parameters for the plots are again $\bar{W}=0.6$, $\bar{D}=1.2$, and $\bar{G}=3$ for both GWFELs. A current of $5 \mathrm{~mA}$ and beam diameters of $d_{x}=d_{y}=150 \mu \mathrm{m}$ are used in the calculation. The gain of the first even mode of the symmetric GWFEL [dashed line, corresponding to curve $C$ in Fig. 6(b)] is about 10 times that of the gains of the two lowest modes of the staggered GWFEL plotted as solid and dotted lines, corresponding to curves $C^{\prime}$ and $D^{\prime}$, respectively, in Fig. 6(a). The gain of a staggered GWFEL is lowered because its axial field (refer to Fig. 7) is weaker than the pure even-mode field (see Fig. 3). In Fig. 8, the

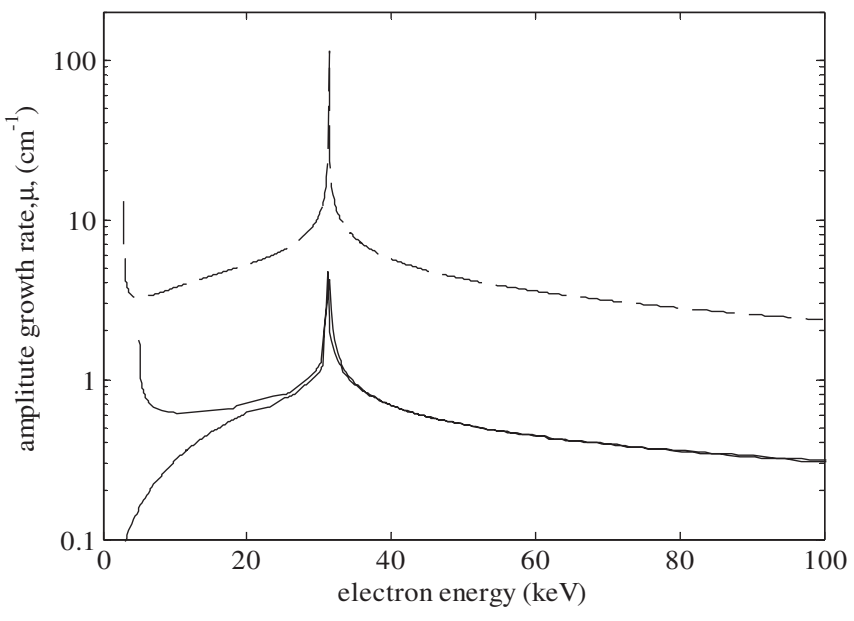

FIG. 8. Gain coefficient of symmetric (dashed line) and staggered (solid and dotted lines) GWFELs versus electron-beam energy. The dashed, solid, and dotted lines are associated with Curves $A, C^{\prime}, D^{\prime}$ in Fig. 6, respectively. The driving current in this calculation is $5 \mathrm{~mA}$ uniformly distributed over beam diameters of $d_{x}=d_{y}=150 \mu \mathrm{m}$. The gain coefficient of a staggered GWFEL is about 10 times lower than that of a symmetric GWFEL of the same structure dimensions.

two gain peaks separated by $0.38 \mathrm{keV}$ reveals detuning at $\bar{k}_{z}=1 / 2$ for the staggered GWFEL.

\section{DISCUSSION AND CONCLUSION}

The proposed GWFEL has a slow-wave structure that is similar to a corrugated cylindrical waveguide used in a millimeter-wave BWO. The GWFEL consists of two planar gratings as its wave-guiding structure. The planar design is a simpler configuration for implementing a radiation source at $\mathrm{THz}$ frequencies.

A GWFEL can function as a BWO in the negative-slope portion of the dispersion curve. Numerical calculations indicate that a GWFEL driven by keV electrons can reach the threshold of backward-wave oscillation with structural parameters of a few hundred micrometers and a beam current of a few $\mathrm{mA}$. The structural loss, while depending on modes and materials, affects the starting current for backward-wave oscillation. Although the calculation did not specifically take into account the structural loss, the electron-beam parameters for establishing oscillation in a lossless GWFEL do not substantially differs from those in a practical sub-THz BWO [14].

At the zero-group-velocity point of a dispersion curve, a BWO is known to have a low start current [15]. These zerogroup-velocity points that appear at $\bar{k}_{z}=m / 2, m=$ $1,2,3, \ldots$ are the Bragg resonances of a periodic structure. At $\bar{k}_{z}=0.5$, a BWO is said to operate with a " $\pi$ " mode, in which the period of the electromagnetic field is twice the period of the structure. The $\pi$ mode operation of a GWFEL can be identified from the mode fields in Figs. 3 and 7. In fact, the zero group velocity is a consequence of a standing 
wave that is established from the distributed feedback of the periodic structure. At the Bragg resonance, the distributed feedback from the corrugation of the structure is resonantly enhanced in both longitudinal directions. A $\pi$-mode GWFEL is analogous to the so-called distributed-feedback laser in solid-state photonics. The forward- and backward-propagating fields in such a distributed-feedback oscillator are related by a coupledwave equation, from which one can find the resonance and threshold conditions subject to a finite loss in the structure and a laser gain from the driving current. This travelingwave model can be applied to prevent the divergence of the loss coefficient due to the zero group velocity at the Bragg resonance [6]. This technique has been used to model all the distributed-feedback lasers in solid-state photonics [16] and was recently applied to a Smith-Purcell FEL operating at Bragg resonance [12]. Although we plan to report the detailed modeling of a distributed-feedback GWFEL in the near future, the gain curves of a GWFEL in Figs. 4 and 8 already clearly show the Bragg resonances. Since a distributed-feedback laser has the advantage of single-longitudinal-mode oscillation, we also expect the GWFEL to be a promising single-frequency radiation source at $\mathrm{THz}$ frequencies.

Unlike a microwave BWO in a cylindrical enclosure, a GWFEL is formed by two planar gratings whose relative position can be flexibly adjusted. Consequently, the dispersion relationship and thus the radiation frequency of a GWFEL can be tuned by adjusting the waveguide gap or the longitudinal displacement of the two gratings. Figures 5 and 6 demonstrate this tuning flexibility of a GWFEL.

In a symmetric GWFEL, the two gratings are transversely aligned to form a mirror image about the $y=0$ plane. The mode profiles of the longitudinal field in a symmetric GWFEL can be divided into two categories, one with even symmetry and one with odd symmetry about the $y=0$ plane. The even-symmetry mode provides appreciable gain to a symmetric GWFEL, whereas the oddsymmetry mode provides almost no gain to a symmetric GWFEL.

Shifting one grating relative to the other in the longitudinal direction also tunes the dispersion curves of a GWFEL. The maximum longitudinal shift is half of the grating period. Interestingly, this half-period displacement can effectively double the spatial frequency of the structure that is seen by the electromagnetic wave, if the waveguide gap is small relative to the wavelength. Consequently, the odd-order Bragg resonances appearing in a symmetric GWFEL are slightly detuned in a staggered GWFEL. Symmetry breaking permits no pure even or pure odd radiation mode in a staggered GWFEL. As a result, the axial field in a staggered GWFEL is generally weaker than the even-mode field in a symmetric GWFEL. The FEL gain of a staggered GWFEL is therefore smaller than that for a symmetric GWFEL operating with an even-mode field.

\section{ACKNOWLEDGMENTS}

The authors would like to thank the National Science Council for financially supporting this research under Contract No. NSC-2112-M-007-027-MY2 and by the NTHU Frontier Research Projects under Code 95N2509E1.

\section{APPENDIX A}

The electric and magnetic fields are continuous at the boundaries $y= \pm G / 2$. Equations (3) and (4) are equated to (9) and (10) at $y=G / 2$ and to (12) and (13) at $y=$ $-G / 2$ to obtain the following expressions:

$$
\begin{gathered}
\sum_{p=-\infty}^{\infty} E_{p}^{t} \sinh \left(\alpha_{p} G\right) \exp \left(-j p \mathrm{~K}_{g} z\right) \exp \left(-j k_{z} z\right)=-\sum_{n=0}^{\infty} \bar{E}_{n}^{t} \cos \left(\frac{n \pi z}{W}\right) \tanh \left(\kappa_{n} D\right), \\
\sum_{p=-\infty}^{\infty}\left[H_{p}^{b} \exp \left(-i \phi_{p}\right)+H_{p}^{t} \cosh \left(\alpha_{p} G\right)\right] \exp \left(-j p \mathrm{~K}_{g} z\right) \exp \left(-j k_{z} z\right)=\sum_{n=0}^{\infty} \bar{H}_{n}^{t} \cos \left(\frac{n \pi z}{W}\right) \operatorname{coth}\left(\kappa_{n} D\right), \\
\sum_{p=-\infty}^{\infty} E_{p}^{b} \sinh \left(\alpha_{p} G\right) \exp \left(-j \phi_{p}\right) \exp \left(-j p \mathrm{~K}_{g} z\right) \exp \left(-j k_{z} z\right)=\sum_{n=0}^{\infty} \bar{E}_{n}^{b} \cos \left[\frac{n \pi(z-d)}{W}\right] \tanh \left(\kappa_{n} D\right), \\
\sum_{p=-\infty}^{\infty}\left[H_{p}^{b} \cosh \left(\alpha_{p} G\right) \exp \left(-j \phi_{p}\right)+H_{p}^{t}\right] \exp \left(-j p \mathrm{~K}_{g} z\right) \exp \left(-j k_{z} z\right)=\sum_{n=0}^{\infty} \bar{H}_{n}^{b} \cos \left[\frac{n \pi(z-d)}{W}\right] \operatorname{coth}\left(\kappa_{n} D\right) .
\end{gathered}
$$

To extract the complex amplitudes of the spatial harmonics, (A1) and (A3) are multiplied by $\exp \left[j\left(q \mathrm{~K}_{g}+k_{z}\right) z\right]$ and integrated over the range $\left[0, \Lambda_{g}\right]$ in $z$ and (A2) and (A4) are multiplied by $\cos (m \pi z / W)$ and integrated over $[0, W]$ in $z$. The following four expressions are thus obtained: 


$$
\begin{aligned}
& \Lambda_{g} E_{q}^{b} \sinh \left(\alpha_{q} G\right) \exp \left(-j \phi_{q}\right)=\sum_{n=0}^{\infty} \bar{E}_{n}^{b} \tanh \left(\kappa_{n} D\right) \Gamma_{q n}^{b}, \\
& \Lambda_{g} E_{q}^{b} \sinh \left(\alpha_{q} G\right)=-\sum_{n=0}^{\infty} \bar{E}_{n}^{t} \tanh \left(\kappa_{n} D\right) \Gamma_{q n}^{t}, \\
& \frac{W\left(1+\delta_{m 0}\right)}{2} \bar{H}_{m}^{b} \operatorname{coth}\left(\kappa_{m} D\right) \\
& =\sum_{p=-\infty}^{\infty}\left[H_{p}^{t}+H_{p}^{b} \cosh \left(\alpha_{p} G\right) \exp \left(-j \phi_{p}\right)\right] \Gamma_{p m}^{b}, \\
& \frac{W\left(1+\delta_{m 0}\right)}{2} \bar{H}_{m}^{t} \operatorname{coth}\left(\kappa_{m} D\right) \\
& =\sum_{p=-\infty}^{\infty}\left[H_{p}^{t} \cosh \left(\alpha_{p} G\right)+H_{p}^{b} \exp \left(-j \phi_{p}\right)\right] \Gamma_{p m}^{t},
\end{aligned}
$$

where $\delta_{i j}$ is the Kronecker delta function, $\Gamma_{p n}^{t}=$ $\exp \left(-j \phi_{p}\right) \Gamma_{p n}^{b}$, and

$$
\begin{aligned}
\Gamma_{p n}^{b}= & \exp \left(-j \phi_{p}\right) \frac{-j\left(p \mathrm{~K}_{g}+k_{z}\right)}{\left(p \mathrm{~K}_{g}+k_{z}\right)^{2}-\left(\frac{n \pi}{W}\right)^{2}} \\
& \times\left\{(-1)^{n} \exp \left[-j\left(p \mathrm{~K}_{g}+k_{z}\right) W\right]-1\right\} .
\end{aligned}
$$

The electric field and magnetic field are linked by Ampere's law, given by

$$
\begin{gathered}
\alpha_{p} H_{p}^{t}=j \omega \varepsilon_{p} E_{p}^{t}, \\
-\alpha_{p} H_{p}^{b}=j \omega \varepsilon_{p} E_{p}^{b}, \\
\kappa_{n} \bar{H}_{n}^{t}=-j \omega \varepsilon_{0} \tanh \left(\kappa_{n} D\right) \bar{E}_{n}^{t},
\end{gathered}
$$

and

$$
\kappa_{n} \bar{H}_{n}^{b}=-j \omega \varepsilon_{0} \tanh \left(\kappa_{n} D\right) \bar{E}_{n}^{b},
$$

where $\varepsilon_{p}=\varepsilon_{0}\left(1+\chi_{p}^{\prime}\right)$ is the permittivity of the plasma dielectric with the electric susceptibility expressed by

$$
\chi_{p}{ }^{\prime}=-\frac{\omega_{p l}^{2}}{\gamma^{3}\left[\omega-c \beta\left(k_{z}+p \mathrm{~K}_{g}\right)\right]^{2}} .
$$

In Eq. (A14) $\gamma$ is the Lorentz factor and $\omega_{p l}$ is the plasma frequency in the laboratory frame.

We first replaced the left-hand side of Eq. (A7) with Eq. (A12) and substituted Eqs. (A12) and (A11) into the

right-hand side of Eq. (A7). We further used Eqs. (A5) and (A6) in the resulting equation to obtain a set of homogeneous linear equations for the field coefficients of the waveguide modes:

$$
\bar{E}_{n}^{b}=\sum_{m=0}^{\infty} a_{n m} \bar{E}_{m}^{t}+b_{n m} \bar{E}_{m}^{b}
$$

and

$$
\bar{E}_{n}^{t}=\sum_{m=0}^{\infty} b_{n m} \bar{E}_{m}^{t}+a_{n m}{ }^{*} \bar{E}_{m}^{b}
$$

where

$$
\begin{aligned}
a_{n m}= & \frac{-2 \kappa_{n}}{W \Lambda_{g}\left(\delta_{n 0}+1\right)} \tanh \left(\kappa_{m} D\right) \sum_{p=-\infty}^{\infty} \frac{1+\delta_{p 0} \chi_{p}^{\prime}}{\alpha_{p} \sinh \left(\alpha_{p} G\right)} \\
& \times \exp \left(j \phi_{p}\right) \Gamma_{p n}^{t} \Gamma_{p m}^{t},
\end{aligned}
$$

and

$$
\begin{aligned}
b_{n m}= & \frac{-2 \kappa_{n}}{W \Lambda_{g}\left(\delta_{n 0}+1\right)} \tanh \left(\kappa_{m} D\right) \sum_{p=-\infty}^{\infty} \frac{1+\delta_{p 0} \chi_{p}^{\prime}}{\alpha_{p} \sinh \left(\alpha_{p} G\right)} \\
& \times \cosh \left(\alpha_{p} G\right) \Gamma_{p n}^{t} \Gamma_{p m}^{t} .
\end{aligned}
$$

In Eqs. (A17) and (A18), the subscripts $m$ and $n$ denote the space harmonics of the modes in the groove region. The matrix representations $\mathbf{A}=\left[a_{m n}\right]$ and $\mathbf{B}=\left[b_{m n}\right]$ can be used to recast Eqs. (A15) and (A16) into the form

$$
\left\{\begin{array}{l}
\overline{\mathbf{E}}^{\mathbf{b}}=\mathbf{A} \overline{\mathbf{E}}^{\mathbf{t}}+\mathbf{B} \overline{\mathbf{E}}^{\mathbf{b}} \\
\overline{\mathbf{E}}^{\mathbf{t}}=\mathbf{B} \overline{\mathbf{E}}^{\mathbf{t}}+\mathbf{A}^{*} \overline{\mathbf{E}}^{\mathbf{b}}
\end{array}\right.
$$

The determinant of the coefficient matrix of the linear system (A19) must vanish to yield a nontrivial solution for the electric field. Accordingly, the characteristic equation of the mode fields is obtained:

$$
\operatorname{det}\left\{(\mathbf{I}-\mathbf{B})^{-1} \mathbf{A}(\mathbf{I}-\mathbf{B})^{-1} \mathbf{A}^{*}-\mathbf{I}\right\}=0,
$$

where I is the identity matrix with sufficient dimensionality. When the wavelength is much smaller than the groove width, the short-circuit approximation [17] can be employed, with which $\overline{\mathrm{E}}_{n \neq 0}^{b}$ and $\overline{\mathrm{E}}_{n \neq 0}^{t}$ are all negligible by comparison with the terms with $n=0$. The characteristic equation is reduced to two terms

$$
b_{00} \pm\left|a_{00}\right|=1,
$$

where

$$
\begin{aligned}
\left|a_{00}\right|= & |\bar{\omega} \bar{W} \tan (2 \pi \bar{\omega} \bar{D})|\left\{\left[\sum_{p=-\infty}^{\infty}\left(1+\delta_{p 0} \chi_{p}^{\prime}\right) \frac{\operatorname{sinc}^{2}\left[\left(p+\bar{k}_{z}\right) \bar{W}\right]}{\bar{\alpha}_{p} \sinh \left(2 \pi \bar{\alpha}_{p} \bar{G}\right)} \cos (2 \pi p \bar{d})\right]^{2}\right. \\
& \left.+\left[\sum_{p=-\infty}^{\infty}\left(1+\delta_{p 0} \chi_{p}^{\prime}\right) \frac{\operatorname{sinc}^{2}\left[\left(p+\bar{k}_{z}\right) \bar{W}\right]}{\bar{\alpha}_{p} \sinh \left(2 \pi \bar{\alpha}_{p} \bar{G}\right)} \sin (2 \pi p \bar{d})\right]^{2}\right\}^{1 / 2} .
\end{aligned}
$$


Equation (A20) is a general expression for describing the dispersion relationship of the electromagnetic wave in a grating waveguide with an arbitrary longitudinal displacement $\bar{d}$ between the two grating halves. Equation (A21) was determined numerically to be accurate within $1 \%$ of Eq. (A20) as long as the groove width of the grating does not exceed $27 \%$ of the wavelength.

\section{APPENDIX B}

In Fig. 6, the odd-order Bragg resonances in a symmetric grating waveguide are detuned in a staggered grating waveguide. The gain coefficient of a staggered GWFEL can be calculated from Eq. (17), and is given by

$$
g=\left.\operatorname{Im}(\delta k)\right|_{\delta \omega=0} \propto \frac{\sqrt{3}}{2} \sqrt[3]{\left|\frac{S\left(\omega_{0}, k_{0}\right)}{R_{\omega}\left(\omega_{0}, k_{0}\right)}\right|} .
$$

Equation (B1) diverges near the Bragg resonance, as shown in Fig. 8. Since Eq. (B1) diverges when $R_{\omega}\left(\omega_{0}, k_{0}\right)$ vanishes, we adopt a perturbation approach and set

$$
R_{\omega}\left(\omega_{0}, \bar{k}_{z}+\Delta \bar{k}_{z}\right)=0,
$$

where $\bar{k}_{z}=m / 2, m=1,2,3 \ldots$ and $\Delta \bar{k}_{z}$ is a small detuning from the resonance. The solution to Eq. (B2) leads to the form

$$
\Delta \bar{k}_{z}= \pm \frac{\sum_{p=-\infty}^{\infty} \frac{\cos ^{p}(m \pi)(p+1 / 2)}{\bar{\alpha}_{p} \sinh \left(2 \pi \bar{\alpha}_{p} \bar{G}\right)}}{f(\bar{G}, \bar{\omega})},
$$

where $f(\bar{G}, \bar{\omega})$ is a collection of the first-order coefficients from the expansion

$$
R^{\prime}\left(\bar{\omega}_{0,} \bar{k}_{z}=m / 2+\delta \bar{k}_{z}, \bar{d}=0.5\right) .
$$

Near the Bragg resonance,

$$
\bar{\alpha}_{p}=\bar{\alpha}_{-(p+1)},
$$

in Eq. (6) when the electron beam is absent. Substituting Eq. (B5) into Eq. (B3) yields

$$
\Delta \bar{k}_{z}= \pm \frac{1}{f(\bar{G}, \bar{\omega})} \sum_{p=0}^{\infty} \frac{\cos ^{p}(m \pi)(p+1 / 2)+\cos ^{-(p+1)}(m \pi)[-(p+1)+1 / 2]}{\bar{\alpha}_{p} \sinh \left(2 \pi \bar{\alpha}_{p} \bar{G}\right)}
$$

The numerator of Eq. (B6) is zero when $m$ is even and nonzero when $m$ is odd. Therefore, detuning occurs only near an odd-order Bragg resonance. The term, $f(\bar{G}, \bar{\omega})$, in the denominator monotonically increases with the waveguide gap $\bar{G}$ and the frequency $\bar{\omega}$. Hence, the detuning decreases rapidly for large $\bar{G}$ or large $\bar{\omega}$.

[1] S. J. Smith and E. M. Purcell, Phys. Rev. 92, 1069 (1953).

[2] E. M. Marshall, P.M. Phillips, and J.E. Walsh, IEEE Trans. Plasma Sci. 16, 199 (1988).

[3] J. H. Killoran, F. L. Hacker, and J. E. Walsh, IEEE Trans. Plasma Sci. 22, 530 (1994).

[4] J. Urata, M. Goldstein, M.F. Kimmitt, A. Naumov, C. Platt, and J. E. Walsh, Phys. Rev. Lett. 80, 516 (1998).

[5] H.L. Andrews, C.H. Boulware, C.A. Brau, and J.D. Jarvis, Phys. Rev. ST Accel. Beams 8, 050703 (2005).

[6] K.-J. Kim and S.-B. Song, Nucl. Instrum. Methods Phys. Res., Sect. A 475, 158 (2001).

[7] L. Schächter and A. Ron, Phys. Rev. A 40, 876 (1989).

[8] C. S. Liu and V. K. Tripathi, IEEE J. Quantum Electron. 35, 1386 (1999).
[9] Charles H. Boulware, Heather L. Andrews, Jonathan D. Jarvis, and Charles A. Brau, in Proceedings of the 28th International Free Electron Laser Conference, BESSY, Berlin, 2006.

[10] H.L. Andrews and C.A. Brau, Phys. Rev. ST Accel. Beams 7, 070701 (2004).

[11] H. Kogelnik and C. V. Shank, J. Appl. Phys. 43, 2327 (1972).

[12] D. Kipnis, E. Dyunin, and A. Gover, in Proceedings of the FEL 2005 Conference, Stanford, CA, JACoW/eConf C0508213 (2005)), pp. 328-330.

[13] John. A. Swegle, Phys. Fluids 30, 1201 (1987).

[14] Millimeter and Submillimeter Wave for Spectroscopy of Solid, edited by G. Grüner, Topics in Applied Physics Vol. 4 (Springer-Verlag, Berlin, 1998).

[15] S. M. Miller, T.M. Antonsen, Jr., B. Levush, A. Bromborsky, D. K. Abe, and Y. Carmel, Phys. Plasmas 1, 730 (1994).

[16] See, for example, G. P. Agrawal and N. K. Dutta, LongWavelength Semiconductor Lasers (Van Nostrand Reinhold, New York, 1986), p. 292.

[17] R.E. Collin, Foundation for Microwave Engineering (McGraw-Hill, New York, 1992), p. 575. 